\title{
A cis-regulatory change underlying the motor neuron-specific loss of terminal selector gene expression in immotile tunicate larvae
}

\author{
Elijah K. Lowe ${ }^{1,2}$, Claudia Racioppi ${ }^{2,3}$, Nadine Peyriéras ${ }^{2,4,5}$, Filomena Ristoratore ${ }^{2,6}$, Lionel \\ Christiaen ${ }^{2,3}$, Billie J. Swalla ${ }^{2,7,8}$, \& Alberto Stolfi ${ }^{1,2 *}$ \\ 1. School of Biological Sciences, Georgia Institute of Technology, Atlanta, GA, USA. \\ 2. Station Biologique de Roscoff, Roscoff, France. \\ 3. Center for Developmental Genetics, Department of Biology, New York University, New York, NY, USA. \\ 4. UPS3611 Complex Systems Institute Paris Île-de-France (ISC-PIF), CNRS, Paris, France. \\ 5. USR3695 BioEmergences, CNRS, Paris-Saclay University, Gif-sur-Yvette, France. \\ 6. Biology and Evolution of Marine Organisms, Stazione Zoologica Anton Dohrn, Naples, Italy. \\ 7. Department of Biology, University of Washington, Seattle, WA, USA. \\ 8. Friday Harbor Laboratories, University of Washington, Friday Harbor, WA, USA. \\ * Corresponding author: alberto.stolfi@biosci.gatech.edu
}

\begin{abstract}
The evolutionary history of animal body plans cannot be fully reconstructed without considering the roles of both novelties and losses. Some of the more remarkable examples of massively parallel evolutionary losses in animals comes from many species in the tunicate genus Molgula that have independently lost the swimming larva and instead develop as tail-less, immotile larvae that bypass the period of swimming and dispersal observed in other tunicates, marine invertebrate chordates that alternate between motile larval and sessile adult life cycle stages. The larvae of Molgula occulta and other tail-less species do not fully develop structures that are essential for swimming behavior, including notochord, tail muscles, and otolith, and loss-of-function mutations have been identified in various genes required for the differentiation of these tissues. However, little is known about the extent of development of the larval nervous system in M. occulta. While differentiated neurons might in principle be entirely dispensable to the non-swimming larva, the adult has a fully functional nervous system like any other tunicate. To further investigate this conundrum, we studied the specification and patterning of the M. occulta Motor Ganglion, which is the key central nervous system compartment that drives the motor movements of swimming tunicate larvae. We found that the expression patterns of important regulators of MG neuron subtype specification are highly conserved during the development of the non-swimming larvae of $M$. occulta, suggesting that the gene networks regulating their expression are largely intact in this species, despite the loss of swimming ability. However, we identified a $M$. occultaspecific reduction in expression of the important motor neuron terminal selector gene Ebf (Collier/OIf/EBF or COE) in the Motor Ganglion. Although M. occulta Ebf is predicted to encode a fully functional protein, its expression was reduced in developing motor neurons when compared to species with swimming larvae, which was corroborated by measuring allelespecific expression of Ebf in interspecific hybrid embryos produced by crossing $M$. occulta with the closely related swimming species M. oculata. Comparative reporter construct experiments also revealed a specific cis-regulatory sequence change that underlies the reduced expression of $M$. occulta Ebf in motor neurons, but not in other tissues and cell types. This points to a potential mechanism for arresting larval motor neuron differentiation in the non-swimming larvae of this species.
\end{abstract}




\section{$\underline{\text { Introduction }}$}

The evolution of animal body plans has occurred not only through evolutionary novelties, but also through losses both subtle and catastrophic (Albalat and Cañestro, 2016). Although they are defined primarily by the absence of structures, cell types, or genes, the evolutionary loss of these various units of selection can help illuminate their functions. For instance, identifying traits that are retained in one species but lost in a closely related species can reveal which are likely to be under purifying selection and provide a measure of their relative adaptive value under certain conditions, e.g. loss of vision in cave-dwelling organisms (Porter et al., 2003).

Some of the most prominent examples of extensive evolutionary losses come from the tunicates (Denoeud et al., 2010; Huber et al., 2000), marine filter-feeding organisms characterized by a protective layer, or "tunic", made mostly of cellulose (Sasakura, 2018). Phylogenomic analyses suggest that vertebrates are the sister group to the tunicates (Bourlat et al., 2006), and most tunicates have a distinct dispersal phase carried out by swimming "tadpole" type larvae that bear the typical chordate body plan: a rigid notochord flanked by striated paraxial muscles, controlled by a dorsal central nervous system. Some tunicate groups, such as the pelagic salps and pyrosomes, have completely lost this larval phase, while in other groups the evolutionary loss of the swimming larva is an ongoing process, affecting specific aspects of larval development and behavior. This phenomenon is particularly prominent in molgulid tunicates, in which many species have independently lost larval structures important for swimming (Hadfield et al., 1995; Huber et al., 2000). The best studied of these is Molgula occulta, which gives rise to anural (tail-less), nonswimming larvae that metamorphose into juveniles without going through the dispersal period of active swimming observed in urodele (tailed) species (Berrill, 1931).
Underlying this radically divergent, non-swimming larval form is the loss of morphogenesis and differentiation of certain cell types that are dispensable for non-swimming larva, like the notochord, tail muscles, or pigmented sensory organs (Jeffery and Swalla, 1991; Swalla and Jeffery, 1990, 1992). For instance, M. occulta larva muscle cells do not differentiate and the species has even lost certain genes encoding proteins specifically required for muscle function, like muscle actin (Kusakabe et al., 1996). Furthermore, M. occulta lack a pigmented otolith cell, important for gravity sensing in swimming larvae (Jiang et al., 2005; Tsuda et al., 2003), due to the loss of genes encoding functional enzymes required for melanogenesis like Tyrosinase and Tyrosinaserelated protein (Tyrp)(Racioppi et al., 2017). Over 17 species with non-swimming larvae have been described in Molgula, and all 31 species in the closely related molgulid genera Eugyra and Bostrichobranchus appear to have non-swimming larvae. The vast majority of molgulids (>150 species) have not been studied at the larval stage, leaving the possibility that many more non-swimming larvae have independently evolved within the clade (Maliska et al., 2013; Shenkar et al., 2019).

Little is known about the extent of nervous system development in the larvae of $M$. occulta and other species with non-swimming larvae. The typical tunicate larva has a minimal nervous system dedicated to controlling its swimming and settlement behavior in response to sensory cues such as light, gravity, and mechanical stimuli (Jiang et al., 2005; Rudolf et al., 2018; Salas et al., 2018; Zega et al., 2006). The larval nervous system of the tunicate Ciona intestinalis has been completely mapped, revealing 177 neurons in a dorsal central nervous system (CNS) and 54 peripheral sensory neurons distributed throughout the epidermis (Ryan et al., 2016, 2018; Ryan and Meinertzhagen, 2019). With 231 total neurons, the Ciona larval nervous system is one of the smallest known in all of metazoa, and thus an intriguing model for the study of chordate-specific principles of neuronal function and development (Nishino, 2018). 
Given the evolutionary loss of other structures important for swimming (notochord, muscles, otolith), we asked whether any neurodevelopmental processes have been lost during the evolution of M. occulta. To this end, we surveyed the development of the nervous system in $M$. occulta embryos, using species with swimming larvae as a basis for comparison. Using in situ hybridization, RNAseq data, and cross-species transgenic assays, we report that neurodevelopmental gene expression and patterning is unexpectedly conserved in $M$. occulta larvae. Most notably, the Motor Ganglion (MG), the CNS structure that controls the swimming movements of tailed larvae, is also found in tailless larvae (Nishino et al., 2010). However, we uncovered specific cis-regulatory mutations that might underlie the reduced transcriptional activity of the key cholinergic neuron terminal selector gene Ebf (Kratsios et al., 2012) in larval motor neurons. However, unlike Tyrosinase or Tyrp, the Ebf gene has not become pseudogenized in M. occulta, likely due to its requirement for the specification of other neurons and cell types that are still important for the complete life cycle of this species.

\section{Methods}

\section{M. occulta and M. oculata embryo collection}

Gonads were dissected from gravid M. occulta and $M$. oculata adults were collected in August (the only time of the year when gravid individuals can be found there) at Station Biologique in Roscoff, France. Molgula occidentalis adults were collected and shipped by Gulf Specimen Marine Lab (Panacea, FL). Eggs from dissected gonads were fertilized in vitro, dechorionated, and fixed as previously described (Stolfi et al., 2014b).

mRNA probe synthesis, in situ hybridization, and immunostaining

Templates for mRNA in situ hybridization probes were cloned by PCR or SMARTer 3'/5'-RACE (Clontech) from cDNA or genomic DNA (see Supplemental File 1 for details). In vitro transcription of labeled probes was and two-color fluorescent in situ hybridization were performed as previously described (Ikuta and Saiga, 2007; Stolfi et al., 2014b). To detect cilia, larvae were incubated with anti-acetylated $\alpha$-tubulin primary antibody (clone 6-11B-1, Sigma) and AlexaFluor-488 secondary antibody (ThermoFisher) as previously described (Pennati et al., 2015). Cell outlines were counterstained with phalloidin AlexFluor-546 conjugate (ThermoFisher) incubated 1:50 for at least 2 hours prior to final washes, and nuclei were labeled with DAPI during the final wash. Embryos were imaged on upright and inverted epifluorescence microscopes (Leica), or TCS SP5 AOBS and TCS SP8 X scanning point confocal systems (Leica).

\section{Hybrid embryo allele-specific differential expression analyses}

M. occulta and M. oculata genomes (Stolfi et al., 2014b) were improved using Redundans (Pryszcz and Gabaldón, 2016), reads from the initial assemblies (PRJNA253689) were used to improve scaffolding, which had been done to improve the $M$. occidentalis genome and gene models we previously published (Lowe and Stolfi, 2018). Additionally, the M. oculata genome was used as a reference to increase the scaffolding of the $M$. occulta genome. To build gene models, previously sequenced RNAseq reads (Lowe et al., 2014) were mapped using hisat2 (v2.1.0)(Kim et al., 2015), converted to BAM and sorted using SAMTools (v1.5) (Li et al., 2009) and merged with picard tools (v2.0.1) (http://broadinstitute.github.io/picard/). Merged bam files were then assembled using Trinity genome-guide command (v2.6.6)(Grabherr et al., 2011). Assembled transcripts were then translated and filtered using TransDecoder (https://github.com/TransDecoder/TransDecoder/wiki) removing any open-reading frames less than 100 amino acids. To improve on contiguity, transcripts were BLASTed against Ciona robusta predicted proteins from ANISEED (Brozovic et al., 2018), then scaffolded using TransPS (Liu et al., 2014). Default parameters were used for all steps and scripts can be found at https://github.com/elijahlowe/tailed. Genomes and transcriptomes used in this study can be found at https://osf.io/mi3r7/ . For differential 
expression analysis, previously sequenced RNAseq reads from $M$. occulta, $M$. oculata and $M$. occulta $\times M$. oculata hybrid embryos from three different stages (Fodor et al. in preparation; Lowe et al., in preparation)(Lowe et al., 2014) were mapped to the M. occulta or M. oculata Ebf gene models using Salmon v0.6.0 (Patro et al., 2015).

\section{Reporter construct cloning and mutagenesis}

Ebf upstream cis-regulatory sequences were cloned from $M$. occulta and M. oculata genomic DNA (see Supplemental File 1 for sequences). Mutations to convert occulta sequences to oculata-like sequences and vice-versa were generated by synthesizing DNA fragments (Twist Bioscience) and subcloning into the full-length reporter plasmids (see Supplemental File 1 for sequences).

\section{Ciona robusta electroporation}

Ciona robusta (i.e. intestinalis Type A) adults were collected and shipped by M-REP (San Diego, CA). Eggs were obtained from dissected adults and dechorionated, fertilized, electroporated, and fixed as previously described (Christiaen et al., 2009a, b; Stolfi et al., 2014b).

\section{$\underline{\text { Results and discussion }}$}

\section{The nervous system of $M$. occulta larvae}

M. occulta (Figure 1A) and M. oculata (Figure 1B) are closely related species that occur sympatrically off the coast of Brittany, France. In spite of their close genetic similarity and their ability to form interspecific hybrids (Swalla and Jeffery, 1990), the larvae of M. occulta are tail-less and non-swimming (Figure 1C). Given its inability to swim, we asked whether the M. occulta larva has a functional nervous system or whether it has been partially lost in evolution like its vestigial notochord, muscles, and pigment cells. Confocal imaging of phalloidin-stained hatching $M$. occulta larvae revealed that they possess a dorsal hollow neural tube, though it is shortened along the anteriorposterior (A-P) axis due to the lack of an extended tail (Figure 1D). Acetylated tubulin immunohistochemistry revealed ciliated cells lining the neuropore and the shortened caudal neural tube (Figure 1E). In the epidermis, we found that only 4 cells at the very posterior tip of the tail bore long cilia typical of tunicate epidermal sensory cells (Figure 1F). Swimming larvae of tunicate species in several different families have an extended caudal nerve cored lined with numerous ciliated ependymal cells down the length of the tail, and bear numerous sensory neurons with elongated cilia, scattered throughout the epidermis of the head and tail (Figure 1G)(Pasini et al., 2006; Ryan et al., 2018; Torrence and Cloney, 1982; Vorontsova et al., 1997). Taken together, these data indicate that both the CNS and peripheral sensory cells of the $M$. occulta larva are only partially formed in comparison to typical solitary tunicate larvae, consistent with the hypothesis that a fully functional larval nervous system may be dispensable for this species.

To survey CNS development in M. occulta embryos, we performed whole-mount mRNA in situ hybridizations for genes that have been well characterized during CNS development in other tunicates with swimming larvae, notably Ciona spp., Halocynthia roretzi, and Molgula occidentalis. The first candidates we analyzed were Celf3/4/5 (also known as ETR-1) Onecut, and Neurogenin, all relatively broad markers of neuronal fate in swimming tunicate larvae (Figure 2AC)(D'Aniello et al., 2011; Imai et al., 2009; Lowe and Stolfi, 2018; Satou et al., 2001; Yagi and Makabe, 2001). Their mRNA in situ patterns clearly revealed the developing embryonic neural tube around the tailbud stage ( 7.5-9.5 hours post-fertilization, hpf). Although M. occulta embryos are more difficult to orient given their nearly perfect spherical shape, the expression patterns formed the basic outline of the brain and MG, appearing quite conserved relative to the orthologous expression patterns in swimming species, especially $M$. occidentalis (Lowe and Stolfi, 2018).

Unfortunately, we were unable to visualize $M$. occulta neurons in greater detail using fluorescent reporter plasmids, as we have done in Ciona and M. occidentalis (Lowe and Stolfi, 2018; Stolfi and Levine, 2011). This is 
bioRxiv preprint doi: https://doi.org/10.1101/567719; this version posted March 5, 2019. The copyright holder for this preprint (which was not certified by peer review) is the author/funder, who has granted bioRxiv a license to display the preprint in perpetuity. It is made available under aCC-BY-NC-ND 4.0 International license.

Lowe et al., March 012019 - preprint copy - BioRxiv
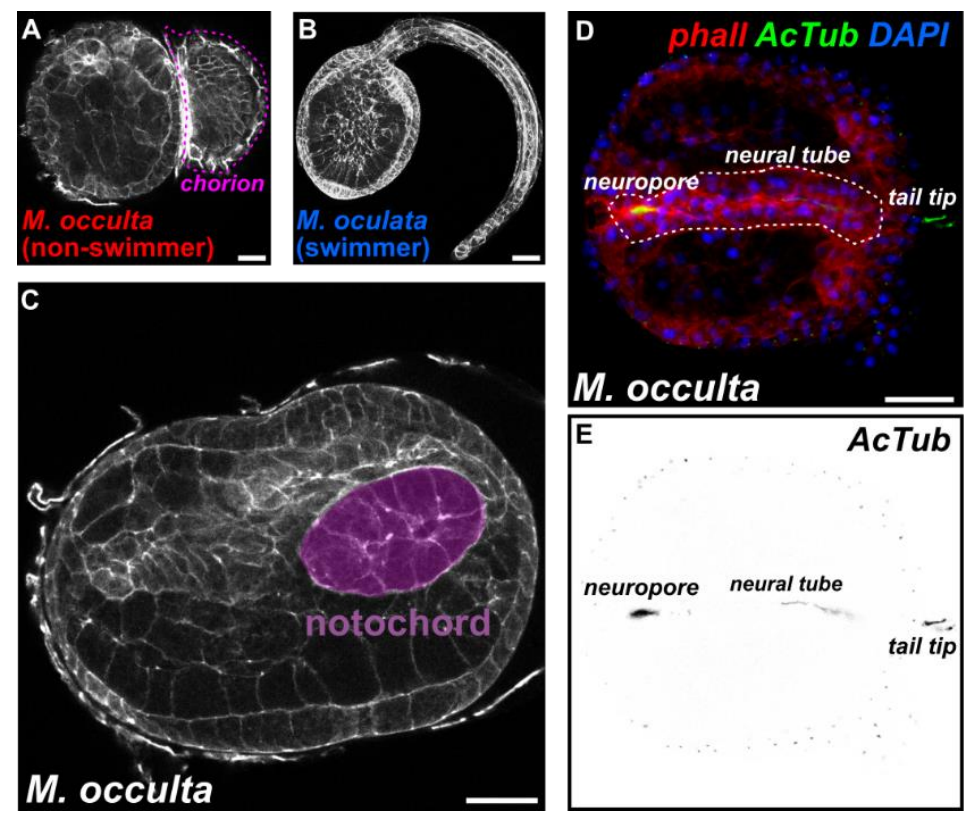
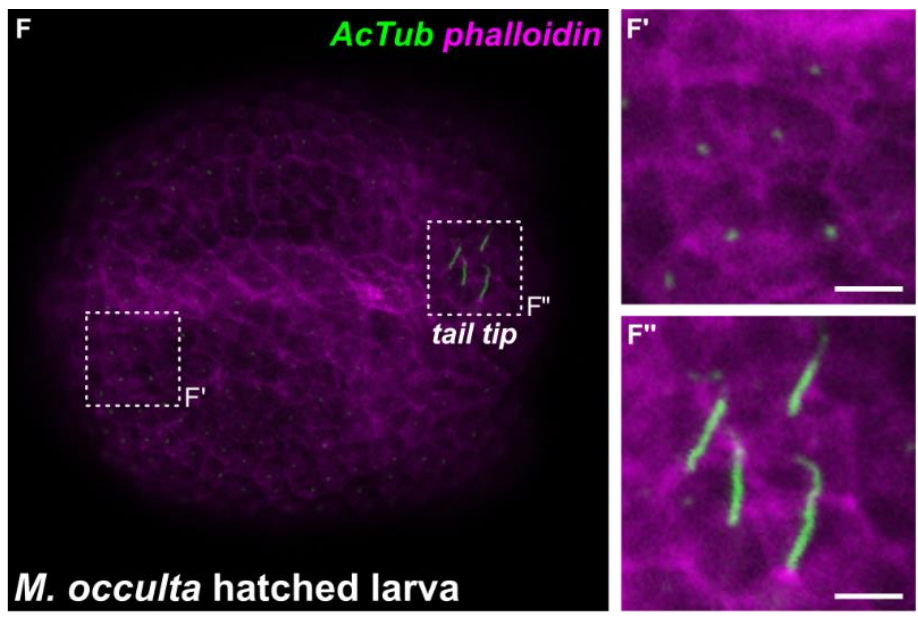

G

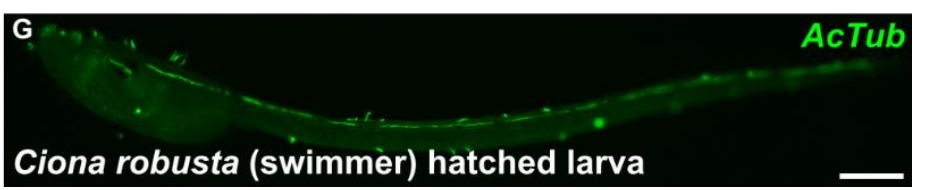

FIGURE 1 - Development and morphology of the non-swimming larvae of Molgula occulta

A) Hatching larva of non-swimming species Molgula occulta, squeezing out of the chorion (pink dotted outline). B) Hatched larva of swimming species Molgula oculata. C) Hatching M. occulta larva showing twenty notochord cells (purple) that fail to undergo convergent extension. D) M. occulta larva stained with phalloidin conjugate (phall, red), anti-acetylated alpha tubulin antibody (AcTub, green), and DAPI (blue), showing presence of acetylated alpha tubulin-rich cilia lining the neuropore/stomodeum and neural tube lumen, and tail tip epidermal cells. E) Inverted monochrome image of acetylated alpha tubulin stain in D). F) M. occulta larva stained with anti-acetylated alpha tubulin antibody (AcTub, green) and phalloidin conjugate (magenta). Inset in $\mathrm{F}^{\prime}$ shows short cilia of epidermal cells covering the majority of the larva. Inset in $\mathrm{F}^{\prime \prime}$ shows longer cilia (presumably sensory) of four tail tip cells. G) Swimming larva of Ciona robusta stained with same anti-acetylated alpha tubulin antibody (AcTub, green), showing abundance of ciliated epidermal sensory cells and neural tube ependymal cells along the entire length of the larva. Scale bars in A-D: $25 \mu \mathrm{m}$. Scale bars in F: $5 \mu \mathrm{m}$. Scale bar in G: $75 \mu \mathrm{m}$.

because $M$. occulta eggs are extremely difficult to fully dechorionate and electroporate. Although we previously reported obtaining a rare electroporated $M$. occulta embryo (Stolfi et al., 2014b), our low success rate and the limited geographic range and spawning season of $M$. occulta/oculata means that we have yet to perform routine transfection of these species. Under these conditions, we proceeded with our analysis using mainly in situ hybridization and nextgeneration sequencing in $M$. occulta embryos, and heterologous reporter construct assays in $C$. robusta embryos.

\section{Gene expression patterns in the $M$. occidentalis Motor Ganglion}

In Ciona, the swimming and escape-response behaviors of the larva are controlled by the MG, a central pattern generator comprised of 22 total neurons (Nishino et al., 2010; Ryan et al., 2016). Within the $M G$, there is a core of 8 left/right pairs of neurons that form the bulk of the synaptic connections within the $M G$, as well as the majority of neuromuscular synapses (Ryan et al., 2016). From now on, we will refer to the core MG neurons on only one side of the larva, with the implicit understanding that our discussion encompasses each left/right pair. We have previously characterized the core cell lineages of the MG in Ciona and M. occidentalis, documenting the highly conserved, invariant specification of each MG neuron subtype and their diagnostic marker gene expression patterns (Imai et al., 2009; Lowe and Stolfi, 2018; Stolfi and Levine, 2011; Stolfi et al., 2011). If the major function of the MG in tunicate larvae is to simply drive swimming behavior, it is likely of little adaptive value and therefore likely to be lost in a non-swimming larva like that of $M$. occulta. On the other hand, MG progenitors might also contribute to the adult nervous system, or differentiated MG neurons might be carrying out other functions beyond swimming, such as triggering metamorphosis. We therefore sought to 
bioRxiv preprint doi: https://doi.org/10.1101/567719; this version posted March 5, 2019. The copyright holder for this preprint (which was not certified by peer review) is the author/funder, who has granted bioRxiv a license to display the preprint in perpetuity. It is made available under aCC-BY-NC-ND 4.0 International license.

Lowe et al., March 012019 - preprint copy - BioRxiv

characterize the expression patterns of candidate regulators of MG patterning in $M$. occulta embryos.

\section{Motor neurons MN1 and MN2}

To ask whether larval motor neurons are specified in M. occulta, we performed in situ hybridization with Mnx, a conserved regulator of motor neuron fate (Ferrier et al., 2001) which in Ciona and M. occidentalis labels the two major motor neurons of the MG, MN1 and MN2 (Ryan et al., 2016). To our surprise, we detected exactly two left/right pairs of cells expressing Mnx in M. occulta tailbud embryos (Figure 2D). This staining appeared identical to Mnx expression in Ciona and $M$. occidentalis, with a gap between the two cells on either side where Interneuron 2 (IN2) should be. This suggests that $M$. occulta embryos specify both major motor neurons found in swimming species.

To possibly distinguish the specification of the two motor neuron subtypes, we did in situ hybridization for Nk6 and Islet, markers specifically for MN1 and MN2 respectively. Nk6 strongly labeled one or two cells on either side of the MG (Figure 2E), similar to its expression in M. occidentalis (Lowe and Stolfi, 2018). These two cells likely correspond to MN1 and IN2, based on their positions and the $M$. occidentalis pattern. In contrast, we were unable to clearly identify MN2 based on Islet expression, as this gene was expressed in a mass of cells in the middle of the embryo, confounding any hope of distinguishing MN2 (Figure 2F). This mass of cells undoubtedly corresponds to notochord cells, which do not intercalate in $M$. occulta (Figure $1 \mathrm{C}$ ). In Ciona and $M$. occidentalis, Islet is also strongly expressed in notochord cells, but because these converge and extend in a very orderly manner, MN2 is clearly distinguishable as an Islet-expressing cell in the developing MG just dorsal to the notochord (Lowe and Stolfi, 2018; Stolfi and Levine, 2011). We were not able to visualize such clear Islet expression in MN2, but we conclude that MN1 and MN2 are indeed specified, based on the combined Nk6 and Mnx expression patterns.
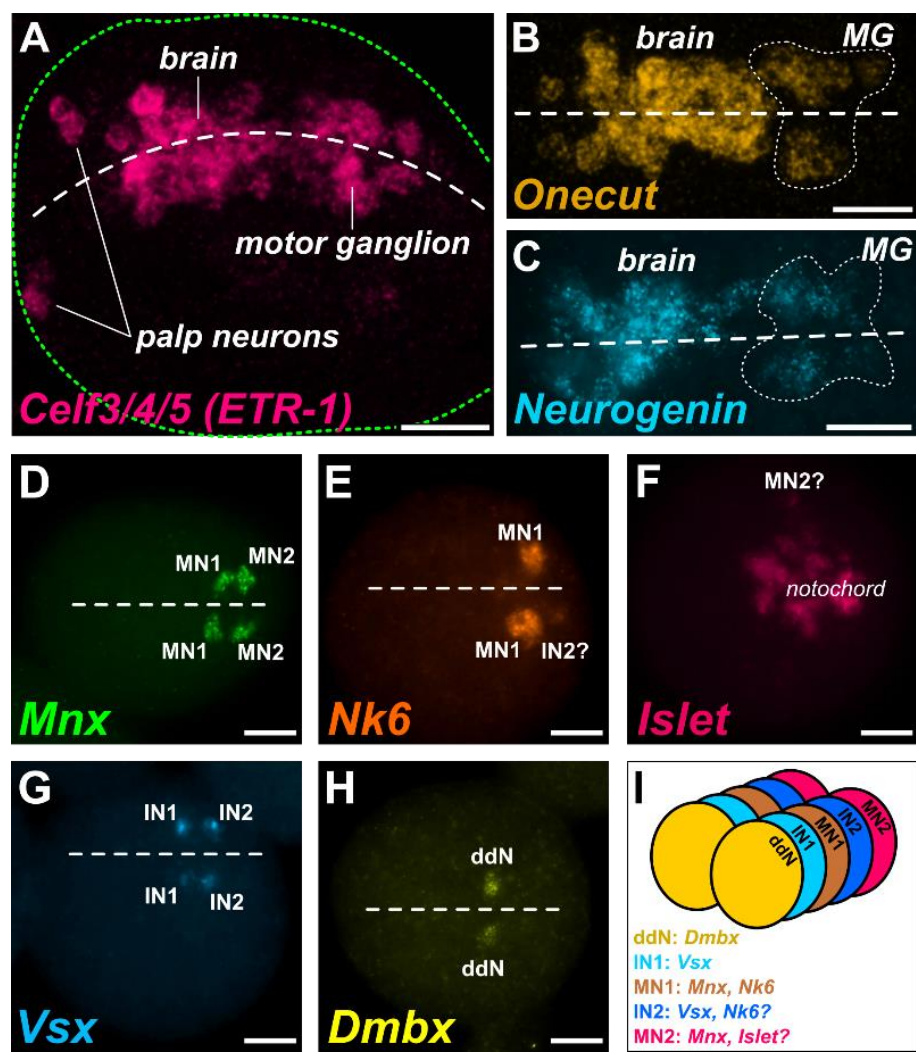

FIGURE 2 - Expression patterns in the developing CNS of Molgula occulta A) Fluorescent whole mount in situ hybridization (ISH) in tailbud embryos for transcripts of neural marker gene Celf3/4/5, also known as ETR-1, showing the developing nervous system including brain, motor ganglion and palps. Embryonic dorsal midline indicated by white dashed line. Embryo outline indicated by green dashed line. B) ISH for neural regulatory gene Onecut, showing brain and motor ganglion (MG)-specific expression. Onecut-expressing MG cells outlined by dotted line. C) ISH for proneural bHLH gene Neurogenin, showing brain and motor ganglion (MG)-specific expression. Neurogenin-expressing MG cells outlined by dotted line. D) ISH for motor neuron-specific marker $M n x$, showing expression marking putative Motor Neuron 1 (MN1) and Motor Neuron 2 (MN2) bilateral pairs. E) ISH for Nk6, showing MN1-specific expression and potentially weak expression in MG Interneuron 2 (IN2) on one side. F) ISH for Islet, showing expression in disorganized notochord cells, and potentially in one MN2 cell. G) ISH for MG interneuron marker gene VsX, showing expression in MG Interneuron 1 (IN1) and MG interneuron 2 (IN2) bilateral pairs. H) ISH for descending decussating neuron (ddN)specific marker Dmbx. I) Cartoon diagram of $M G$ neuron subtype organization in M. occulta based on ISH data and comparisons to the MG of swimming species, namely Ciona robusta and Molgula occidentalis. Dorsal midline in B-E and G-H indicated by dashed line. Scale bars: $25 \mu \mathrm{m}$

\section{MG Interneurons 1 and 2 (MGIN1 and MGIN2)}

In the Ciona MG connectome, three pairs of descending interneurons are predicted to play an important role in both the rhythmicity of swimming movements and their modulation by inputs from the 
sensory vesicle (Kourakis et al., 2019; Ryan et al., 2016; Salas et al., 2018). Of these, the best studied are MGIN1 and MGIN2 (referred to from now on as IN1 and IN2 respectively), which flank MN1. Both are presumed excitatory interneurons that arise from the A9.30 lineage (Cole and Meinertzhagen, 2004). In Ciona and $M$. occidentalis, they are marked by expression of Vsx (Imai et al., 2009; Stolfi and Levine, 2011), the ortholog of conserved spinal cord interneuron regulator $\mathrm{Vsx2/Ch \times 10} \mathrm{(Altun-Gultekin} \mathrm{et}$ al., 2001; Kimura et al., 2006; Liu et al., 1994).

We found that in M. occulta, Vsx labels two cells on either side of the neural tube at the tailbud stage (Figure 2G). This is similar to the orthologous expression patterns in Ciona and $M$. occidentalis. In Ciona, VsX is activated in IN1 and IN2 at two different time points (Stolfi and Levine, 2011). IN2 is the first interneuron to differentiate, and activates Vsx early, before IN1 is even born Later, after IN1 is born and specified, Vsx is activated in this cell. In M. occidentalis, we showed that $V s x$ expression appears concurrently in IN2 and in a progenitor cell that will give rise to IN1, indicating a temporal shift towards precocious activation of VsX in the IN1 lineage (Lowe and Stolfi, 2018). Here, we were unable to ascertain the relative timing of Vsx expression and the identities of the VsXexpressing cells but can conclude that IN1 and IN2 specification is conserved in M. occulta.

\section{Descending decussating neurons}

The descending decussating neuron ( $d d N)$ is a highly unique neuron at the periphery of the core MG in both Ciona and $M$. occidentalis. It is the only descending neuron that decussates, or projects its axon contralaterally (as the name implies). Due to its position and connectivity within the Ciona MG, it was proposed to be the homolog of vertebrate Mauthner cells and to function in a homologous escape response pathway (Ryan et al., 2017; Takamura et al., 2010). In Ciona and $M$. occidentalis, the $d d N$ is marked by expression of Dmbx (Lowe and Stolfi, 2018; Takahashi and Holland, 2004). In M. occulta tailbud embryos, Dmbx was found to be expressed in a single pair of cells
(Figure $2 \mathrm{H}$ ), likely corresponding to the ddNs or their immediate progenitors, the A11.120 cells. We conclude that the $M$. occulta larva specifies ddNs despite being physically incapable of engaging in escape response maneuvers. Taken together, these data reveal that neuronal subtypes are arrayed in the $M$. occulta $M G$ in a manner that is identical to that previously described in swimming species such as $M$. occidentalis and Ciona spp. (Figure 21). This suggests that, in spite of the non-swimming nature of the $M$. occulta larva, MG neuron specification and patterning have not been evolutionarily lost.

\section{Reduced expression of Ebf as a potential mechanism of impaired MG neuron differentiation}

Although developmental patterning of the MG appears to be conserved in the non-swimming larva of M. occulta, we further pursued the hypothesis that perhaps the differentiation of specific MG neurons may not occur in $M$. occulta, similar to how the differentiation (but not the specification) of notochord, tail muscle, and otolith cells have been lost. To address this possibility, we analyzed other genes known to be involved in motor neuron differentiation. One of these is the transcription factor Ebf (also known as Collier/OIf/EBF or COE), a conserved "terminal selector" (Hobert, 2008) for cholinergic motor neurons (Kratsios et al., 2012). In Ciona, Ebf is required for MN2 differentiation (Stolfi et al., 2014a) and is sufficient to activate cholinergic gene expression when ectopically expressed in other cell types (Kratsios et al., 2012). When we looked at Ebf expression in M. occulta, we noticed that it appeared substantially weaker in the MG (but not in other cells) compared to its orthologous expression in $M$. occidentalis, although this assay was not quantitative (Figure $3 A, B$ ). Since Ebf regulates key steps between specification and differentiation in motor neurons, we hypothesized that Ebf expression might have been evolutionarily lost in the vestigial MG of $M$. occulta. To test this further, we turned to a more quantitative interspecies comparison of gene expression. 
bioRxiv preprint doi: https://doi.org/10.1101/567719; this version posted March 5, 2019. The copyright holder for this preprint (which was not certified by peer review) is the author/funder, who has granted bioRxiv a license to display the preprint in perpetuity. It is made available under aCC-BY-NC-ND 4.0 International license.

Lowe et al., March 012019 - preprint copy - BioRxiv

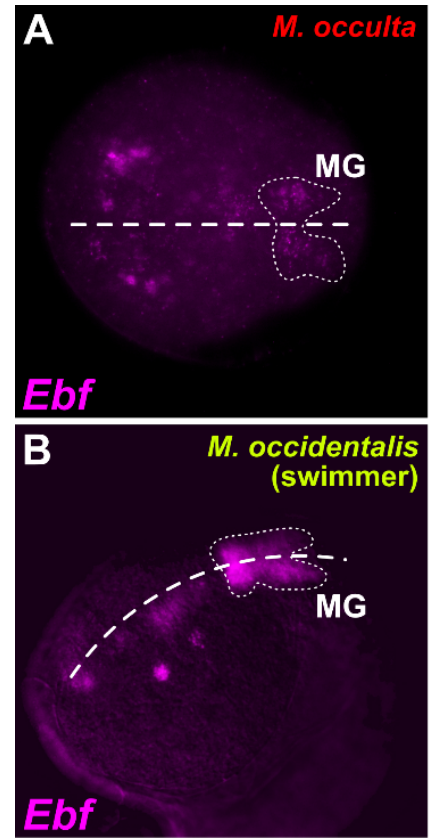

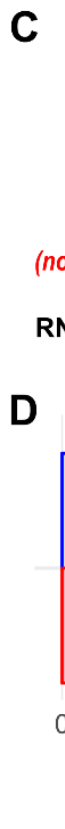
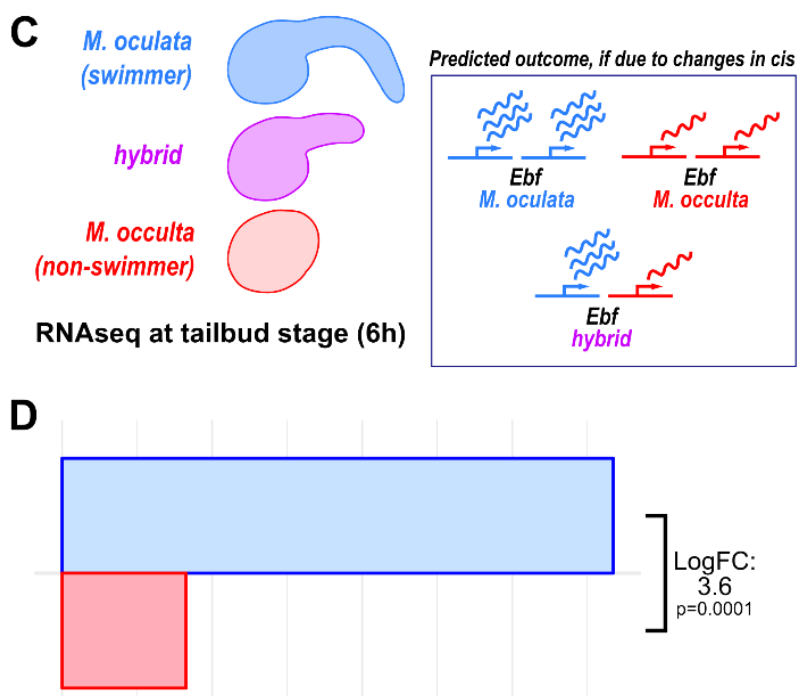

0 50 100 effective Ebf read counts
from 6 hpf hybrids

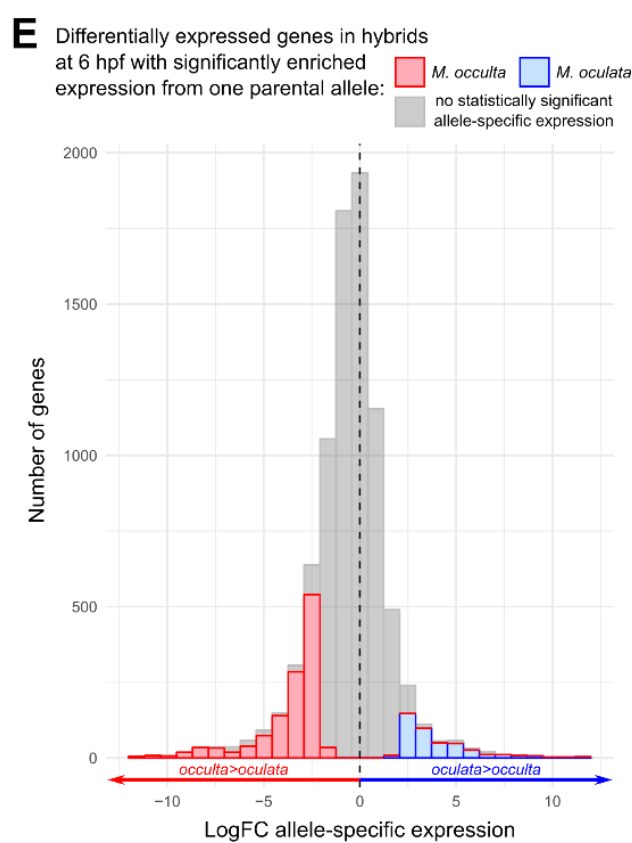

FIGURE 3 - Loss of MG-specific transcriptional activity of the terminal selector gene Ebf in M. occulta

A) Fluorescent whole mount in situ hybridization (ISH) for the Ebf gene in tailbud-stage M. occulta embryo. MG outlined by dotted line, showing relatively weak expression of Ebf, compared to its expression in other cells in the same embryo, and to its expression in swimming species (see panel B). B) ISH for Ebf in tailbud stage embryo of the swimming species Molgula occidentalis, showing strong expression in the MG (dotted outline). C) Diagram of RNAseq analysis of hybrid embryos, produced by fertilizing eggs of non-swimmer M. occulta with sperm from swimmer M. oculata (left). If reduced transcriptional activity of Ebf in $M$. occulta is due to evolutionary changes in cis, the hybrid should show differential allele-specific Ebf expression that recapitulates the differential expression of Ebf observed between the parental species. D) RNAseq read counts from libraries prepared from pooled hybrid embryos at 6 hours post-fertilization ( $\mathrm{hpf}$ ), showing greater effective read count representing the M. oculata (swimmer) Ebf allele relative to the $M$. occulta (non-swimmer) allele, for a $\log _{2} F C$ of $3.6(p=0.0001)$. Embryonic dorsal midline in $A$ and $B$ indicated by dashed line. E) Distribution of genes that are differentially expressed in hybrid embryos at $6 \mathrm{hpf}\left(\log _{2} \mathrm{FC}>1.5, \mathrm{p}<0.05\right)$ according to allele-specific expression, measured and binned by $\log _{2} F C$. Positive values indicate more abundant $M$. oculata parental allele transcripts, negative values indicate more abundant $M$. occulta parental allele transcripts. Colored bars represent those bins showing statistically significant $(p<0.05)$ allele-specific expression, grey bars represent bins showing no such statistically significant expression. While the majority of differentially expressed genes in the hybrid at 6 hpf show no statistically significant allele-specific expression (gray), $64 \%$ of genes with statistically significant allele-specific expression show greater expression from the $M$. occulta parental allele, indicating that enrichment of $M$. oculata Ebf transcripts in hybrids cannot be explained by genome-wide bias towards $M$. oculata parental allele expression.

To compare species-specific transcriptional activity of the Ebf locus, we analyzed RNAseq data from embryos of $M$. occulta, the closely related swimming species $M$. oculata, and their interspecific hybrids (M. occulta egg fertilized with $M$. oculata sperm) (Figure 3C)(Fodor et al., in preparation). When we mapped RNAseq reads obtained from the interspecific hybrid to either parental Ebf allele, we found that transcripts of the $M$. occulta Ebf allele were significantly depleted relative to the M. oculata allele ( $\left.\log _{2} \mathrm{FC}=-3.6, \mathrm{p}=0.0001\right)$ (Figure

3D), even though $64 \%$ of the 8306 genes significantly differentially expressed at 6 hpf actually showed allelespecific expression that was higher for the M. occulta parental allele relative to the $M$. oculata parental allele (Figure 3E) Taken together, these data indicate that evolutionary changes in cis have rendered the $M$. occulta Ebf gene less transcriptionally active in embryonic development.

\section{A cis-regulatory change affecting Ebf expression specifically in MG neurons, but not in other cells}

To test for potential differences in cis that could explain this differential activity of $M$. occulta and $M$. oculata parental Ebf alleles, we constructed reporter plasmids using cis-regulatory sequences upstream of Molgula Ebf genes. Unfortunately, given our inability to electroporate M. occulta/oculata, we turned to $C$. 
A

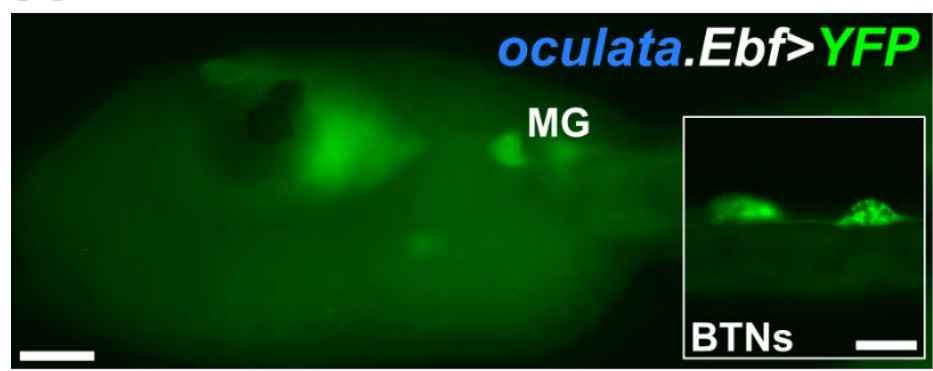

oculata.Ebf>mCherry

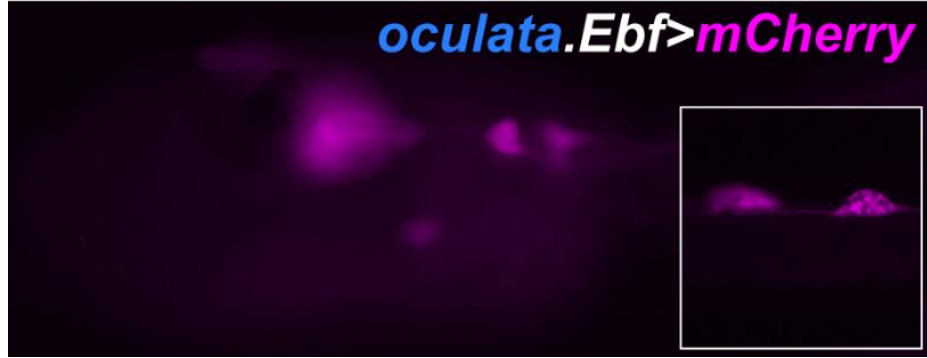

B
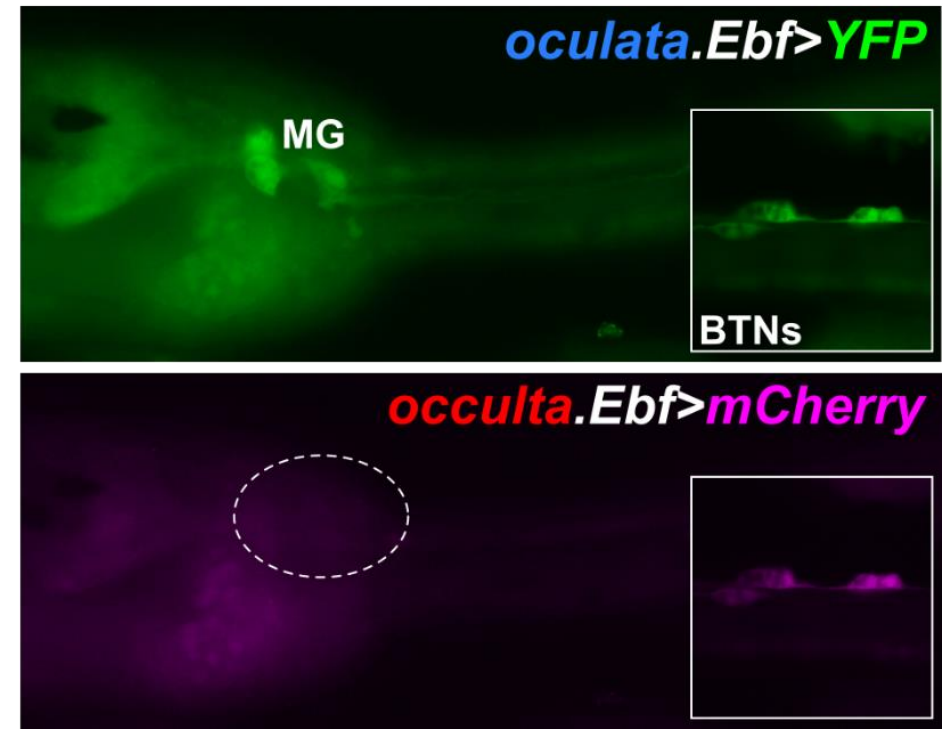

FIGURE 4 - Differential activity of Ebf reporter plasmids in Ciona robusta electroporation assay

A) Ciona robusta larva electroporated with a mixture of M. oculata Ebf>YFP (green, top) and M. oculata Ebf $>m C h e r r y$ (magenta, bottom) plasmids, showing perfect co-expression in the motor ganglion (MG), and in other neurons including Bipolar Tail Neurons (BTNs, insets). B) Ciona robusta larva electroporated with a mixture of M. oculata Ebf>YFP (green, top) and M. occulta Ebf>mCherry (magenta, bottom) plasmids, showing absence of mCherry expression specifically in the MG (region indicated by dashed circle). In contrast, co-expression of YFP and mCherry in BTNs (insets) shows that reporter plasmid activity in other neurons is conserved. Scale bars in A: $25 \mu \mathrm{m}$. All panels and insets are at the same respective scales.

robusta, which can be routinely electroporated in the laboratory with plasmid DNA (Zeller, 2018). When we electroporated $M$. oculata Ebf reporter constructs (oculata.Ebf -3654/+24>XFP) into Ciona embryos, we observed reporter gene expression in MG neurons, Bipolar Tail Neurons (BTNs), and some brain neurons (Figure 4A). Although there is no non-coding sequence conservation between Molgula and Ciona, and acute developmental system drift has rendered many Molgula cis-regulatory sequences inactive in Ciona (Stolfi et al., 2014b), in this case there was enough conservation of the underlying regulatory logic to recapitulate the expression of Ebf in these various Ciona neuronal subtypes.

When we electroporated the corresponding $M$. occulta constructs (occulta.Ebf -3659/+24>XFP), reporter gene expression was observed in all the same neurons except MG neurons (Figure 4B) Thus, our heterologous reporter plasmid assays in Ciona indicated that $M$. occulta-specific cis-regulatory changes might underlie lower levels of Ebf expression in MG neurons, without affecting its expression in other neurons.

To identify the potential cis-regulatory changes underlying this difference in Ebf activation, we aligned $735 \mathrm{bp}$ of non-coding sequence immediately $5^{\prime}$ of $M$. oculata Ebf start codon to the orthologous sequence in M. occulta (Figure 5A). The aligned sequences share $>90 \%$ identity, consistent with the close genetic relationship between these two species. This alignment revealed an unusually divergent sequence motif $\sim 470$ bp upstream of the Ebf translation start codon (Figure 5B). This sequence was of particular interest to us because these changes were observed adjacent to a conserved E-box site that might be a binding site for Neurogenin. In C. robusta, Ebf is activated in the MG by the proneural bHLH factor Neurogenin (Imai et al., 2009). We identified a conserved E-box site in experimentally validated upstream Ebf cis-regulatory sequences isolated from C. robusta, $C$. savignyi, and $M$. occidentalis (Supplemental File 1), even though these sequences show poor overall conservation with the $M$. 
bioRxiv preprint doi: https://doi.org/10.1101/567719; this version posted March 5,2019 . The copyright holder for this preprint (which was not certified by peer review) is the author/funder, who has granted bioRxiv a license to display the preprint in perpetuity. It is made available under aCC-BY-NC-ND 4.0 International license.

Lowe et al., March 012019 - preprint copy - BioRxiv
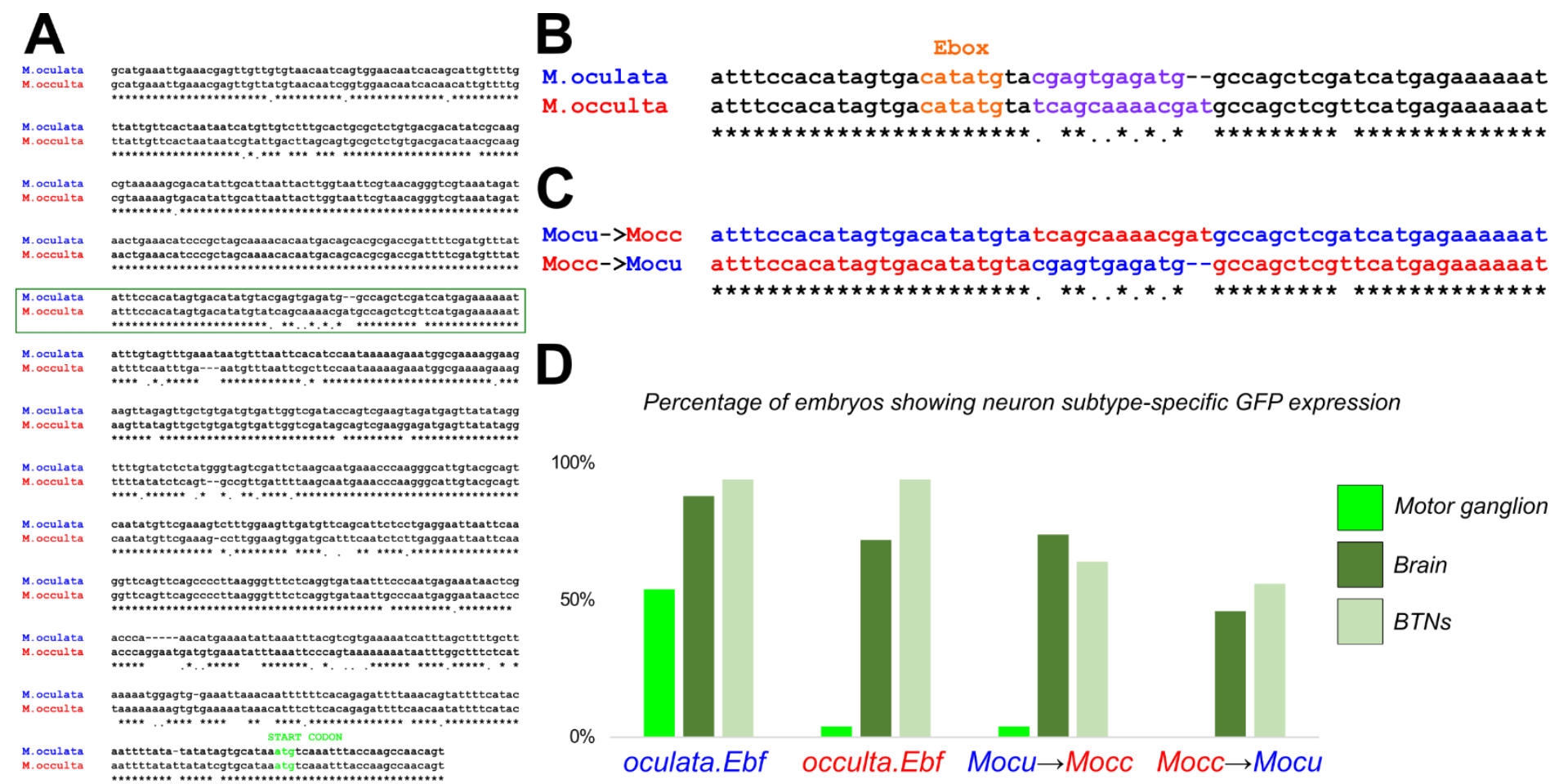

Percentage of embryos showing neuron subtype-specific GFP expression

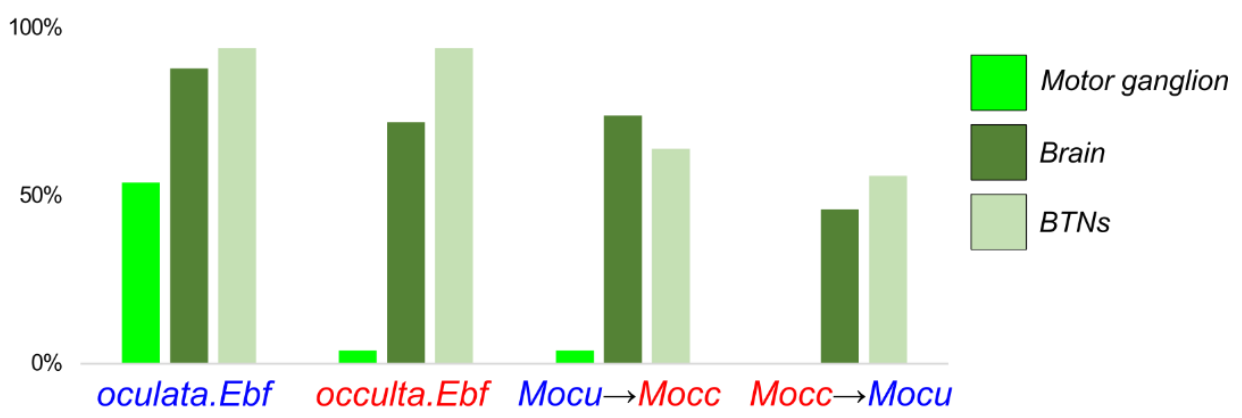

FIGURE 5 - A cis-regulatory change underlying MG-specific loss of Ebf expression in M. occulta

A) Alignment of sequences $5^{\prime}$ upstream of Ebf in M. oculata and M. occulta. B) Sequence from boxed area in A, showing highly conserved sequences including a candidate E-box site potentially bound by Neurogenin, adjacent to a highly divergent motif (purple font). C) Mutated sequences in Mocu $\rightarrow$ Mocc $>$ GFP and Mocc $\rightarrow$ Mocu $>$ GFP constructs in which the highly divergent motif has been swapped between M. oculata and M. occulta Ebf reporter constructs. D) Results of scoring Ciona robusta larvae electroporated with the wild-type parental Ebf reporter plasmids and the mutated "swapped" constructs. Changing the M. oculata divergent motif sequence to the M. occulta sequence results in loss of GFP expression specifically in the motor ganglion, but not in brain or bipolar tail neurons (BTNs). The converse mutation, changing the $M$. occulta motif to resemble the $M$. oculata sequence was not sufficient to restore GFP expression in the motor ganglion. $n=50$ embryos for each construct.

oculata/occulta sequences. The presence of an E-box within 800 bp of the start codon in all tunicate species surveyed suggested this region might be part of a functionally conserved minimal enhancer for MGspecific Ebf regulation. When we compared all 3 Molgula sequences, we found that this E-box-adjacent motif (AGATGGC) was conserved in both $M$. oculata and the more distantly related $M$. occidentalis, but not in $M$. occulta. Because the larvae of $M$. occidentalis are also swimming, and because this $M$. occidentalis Ebf cis-regulatory sequence also drives reporter gene expression in C. robusta MG neurons (Stolfi et al., 2014b), we hypothesized that the E-box-adjacent motif may be crucial for MG-specific expression. Therefore, we decided to test whether the loss of the E-box-adjacent motif might explain the different transcriptional activities of swimming vs. nonswimming Molgula Ebf loci.

To directly test whether $M$. occulta-specific sequence changes are responsible for the loss of $M$. occulta $E b f$ transcription in the MG, we mutated the E-boxadjacent sequence in oculata.Ebf $-3654 /+24>$ GFP to match the $M$. occulta sequence (CGAGTGAGATG to TCAGCAAAACGAT)(Figure 5C). When we electroporated this mutated reporter plasmid (Mocu-$>$ Mocc $>$ GFP) in Ciona, we found that its transcriptional activity was almost completely abolished specifically in the $M G$, but had little effect on reporter gene expression in other neurons (Figure 5D). These data suggest that the mutated motif is necessary for Ebf expression in MG neurons in M. oculata. Alternatively, the $M$. occulta-specific sequence might have created a 
novel binding site for a MG-specific repressor, but the presence of the E-box-adjacent in $M$. occidentalis supports the former explanation. Although we attempted to "resurrect" the putative ancestral sequence by mutating the E-box-adjacent site in occulta.Ebf $-3659 /+24>$ GFP to resemble the $M$. oculata sequence (Mocc-->Mocu>GFP), this was not sufficient to restore activity, hinting at the accumulation of multiple loss-of-function (or compensatory) mutations in other parts of the $M$. occulta Ebf cis-regulatory sequence.

\section{Conclusions}

We have investigated the molecular signatures of MG neuron specification and differentiation in a nonswimming molgulid tunicate larva, finding a surprising conservation of cell-specific expression patterns of key developmental regulators during MG development. This could indicate that at least some MG neurons might be specified and might differentiate in $M$. occulta in spite of the evolutionary loss of the larval tail and swimming behavior. Unfortunately, we were unable to investigate neuronal morphology using reporter plasmids, due to our inability to routinely transfect $M$. occulta embryos. Once this method is established, it will be interesting to revisit this question to fully document the extent of MG development in this species. Nonetheless, MG neuron function is likely seriously impaired in $M$. occulta, given the reduced levels of Ebf expression in $M$. occulta and of the $M$. occulta Ebf allele in interspecific hybrids. In addition to activating the expression of MG neuron-specific regulators like Islet (Stolfi et al., 2014a), Ebf has been shown to act as a conserved "terminal selector" that regulates the terminal differentiation of cholinergic motor neurons throughout Metazoa, including in the tunicate C. robusta (Kratsios et al., 2012). We therefore expect that reduced Ebf expression in the $M$. occulta MG would likely result in similarly reduced or abrogated expression of cholinergic effectors and other cellular machinery required for motor neuron function.
Using cis-regulatory mutations and heterologous reporter plasmid assays, we demonstrate specific changes to conserved cis-regulatory sequences upstream of $M$. occulta Ebf have affected its expression in MG neurons, but not in other neurons or cell types. If these results hold, this would represent a distinct example of tissue-specific gene expression loss in this species. Previously, it has been shown that evolutionary loss of functional larval structures in $M$. occulta correlates with loss-of-function non-coding mutations resulting in the loss of crucial proteins such as muscle actin for tail muscle activity (Kusakabe et al., 1996) or Tyrosinase for melanin pigment synthesis in the gravity-sensing otolith organ (Racioppi et al., 2017). However, these proteins are only expressed in larval structures involved in larval motility (tail muscle cells and otolith, respectively), being apparently dispensable for the development of other larval and adult tissues. In contrast, M. occulta Ebf is not a pseudogene and is predicted to encode a fully functional protein. This difference is likely because Ebf is a regulator that is required for the development of myriad cell types throughout the life cycle of $M$. occulta, especially in the sessile adults of this species, which are morphologically similar to adults of other species, swimming and non-swimming alike. Thus, even if Ebf expression is not needed in MG motor neurons (in the absence of functional larval muscles), its requirement in other tissues may have resulted instead in the evolutionary loss of tissue-specific cisregulatory elements, as our data indicate. It may be that the mechanisms we show here underlying cellspecific loss of Ebf expression in M. occulta are representative of constraints acting on traits that are lost from a particular developmental stage, but that are still retained in a different stage of the same organism's life cycle. Studying these losses and the mechanisms underlying such losses will complement the principles learned from studies of traits that are dispensable to the entire life cycle of a particular species, such as the loss of vision in cave-dwelling organisms. 
Is the evolutionary loss of MG development an ongoing process in M. occulta? Might these processes break down further, as has happened with the loss of genes encoding melanogenesis or muscle structural proteins in this same species? Or are there special constraints that have maintained the neuron subtypespecific marker gene expression we see in the MG? Has MG neuron subtype-specific gene expression persisted because the same regulatory logic is required for expression in adult neurons or other tissues? Alternatively, we know that some MG precursors remain undifferentiated in the larva but can contribute to the adult nervous system (Horie et al., 2011). Perhaps the cell-specific transcription factor expression we observe in the $M$. occulta $M G$ reflects shared mechanisms of patterning both differentiated larval neurons and undifferentiated adult neural precursors. Another intriguing possibility is that the tunicate $M G$ is required for other larval behaviors beyond swimming. For instance, perhaps MG neurons are required for triggering developmental processes during the metamorphosis of the larva into a juvenile. In this scenario, specific MG neuron subtypes might still be necessary for the proper metamorphosis of $M$. occulta larvae, even if Ebf-dependent activation of motor neuron effectors in the MG is not required and might have even been selected against. A deeper understanding of the mechanisms underlying both larval and adult neurogenesis in tunicates, and the role of neural activity in the transition to the adult phase, will be needed to answer these and other questions related to the loss of larval-specific structures in nonswimming species.

\section{Acknowledgments}

We dedicate this manuscript to the memory of Alexander "Zander" Fodor. We thank C. Titus Brown for his encouragement and continued support. We thank Stéphane Hourdez, Sophie Booker, and Xavier Bailly of the Station Biologique for help in carrying out experiments at Roscoff. We thank Susanne Gibboney for assistance with cloning Molgula Ebf reporter plasmids. This work was supported by NIH award R0O
HD084814 to AS, by start-up package from the College or Arts and Science at New York University and $\mathrm{NIH}$ award R01 GM096032 to LC, by France Biolmaging infrastructure ANR-10-INBS-04 to NP, and by a University of Washington Royalty Research Fund award (A118261) to BJS. The collaborative MoEvoDevo network is supported by ASSEMBLE (Association of European Marine Biological Laboratories) MARINE. CR was supported by a long-term fellowship ALTF 16082014 from EMBO and by a travel grant from the Boehringer Ingelheim Fonds. This material is also based in part upon collaborative work by BJS and EKL and C. Titus Brown, supported by the National Science Foundation under Cooperative Agreement No. DBI0939454 BEACON, A Center for the Study of Evolution in Action. Any opinions, findings, and conclusions or recommendations expressed in this material are those of the author(s) and do not necessarily reflect the views of the National Science Foundation.

\section{$\underline{\text { References }}$}

Albalat, R., Cañestro, C.J.N.R.G., 2016. Evolution by gene loss. 17, 379.

Altun-Gultekin, Z., Andachi, Y., Tsalik, E.L., Pilgrim, D., Kohara, Y., Hobert, O., 2001. A regulatory cascade of three homeobox genes, ceh-10, ttx-3 and ceh-23, controls cell fate specification of a defined interneuron class in C. elegans. Development 128, 1951-1969.

Berrill, N.J., 1931. Studies in tunicate development. Part II. Abbreviation of development in the Molgulidae. Philosophical Transactions of the Royal Society of London. Series B, Containing Papers of a Biological Character 219, 281-346.

Bourlat, S.J., Juliusdottir, T., Lowe, C.J., Freeman, R., Aronowicz, J., Kirschner, M., Lander, E.S., Thorndyke, M., Nakano, H., Kohn, A.B., Heyland, A., Moroz, L.L., Copley, R.R., Telford, M.J., 2006. Deuterostome phylogeny reveals monophyletic chordates and the new phylum Xenoturbellida. Nature 444, 85.

Brozovic, M., Dantec, C., Dardaillon, J., Dauga, D., Faure, E., Gineste, M., Louis, A., Naville, M., Nitta, K.R., Piette, J., Reeves, W., Scornavacca, C., Simion, P., Vincentelli, R., Bellec, M., Aicha, S.B., Fagotto, M., Guéroult-Bellone, M., Haeussler, M., Jacox, E., Lowe, E.K., Mendez, M., Roberge, A., Stolfi, A., Yokomori, R., Brown, C T., Cambillau, C., Christiaen, L., Delsuc, F., Douzery, E., Dumollard, R., Kusakabe, T., Nakai, K., Nishida, H., Satou, Y., Swalla, B., Veeman, M., Volff, J.-N., Lemaire, P., 2018. ANISEED 2017: extending the integrated ascidian database to the exploration and evolutionary comparison of genome-scale datasets. Nucleic Acids Research 46, D718D725.

Christiaen, L., Wagner, E., Shi, W., Levine, M., 2009a. Electroporation of transgenic DNAs in the sea squirt Ciona. Cold Spring Harbor Protocols 2009, pdb. prot5345.

Christiaen, L., Wagner, E., Shi, W., Levine, M., 2009b. Isolation of sea squirt (Ciona) gametes, fertilization, dechorionation, and development. Cold Spring Harbor Protocols 2009, pdb. prot5344. 
Cole, A.G., Meinertzhagen, I.A., 2004. The central nervous system of the ascidian larva: mitotic history of cells forming the neural tube in late embryonic Ciona intestinalis. Developmental biology 271, 239-262.

D'Aniello, E., Pezzotti, M.R., Locascio, A., Branno, M., 2011. Onecut is a direct neural-specific transcriptional activator of $\mathrm{Rx}$ in Ciona intestinalis. Developmental biology 355, 358-371.

Denoeud, F., Henriet, S., Mungpakdee, S., Aury, J.-M., Da Silva, C., Brinkmann, H., Mikhaleva, J., Olsen, L.C., Jubin, C., Cañestro, C.J.S., 2010. Plasticity of animal genome architecture unmasked by rapid evolution of a pelagic tunicate. 330, 1381-1385.

Ferrier, D.E., Brooke, N.M., Panopoulou, G., Holland, P.W., 2001. The Mnx homeobox gene class defined by HB9, MNR2 and amphioxus AmphiMnx. Development Genes \& Evolution 211.

Grabherr, M.G., Haas, B.J., Yassour, M., Levin, J.Z., Thompson, D.A., Amit, I., Adiconis, X., Fan, L., Raychowdhury, R., Zeng, Q., Chen, Z., Mauceli, E., Hacohen, N., Gnirke, A., Rhind, N., di Palma, F., Birren, B.W., Nusbaum, C., Lindblad-Toh, K., Friedman, N., Regev, A., 2011. Full-length transcriptome assembly from RNA-Seq data without a reference genome. Nature biotechnology 29, 644-652.

Hadfield, K.A., Swalla, B.J., Jeffery, W.R., 1995. Multiple origins of anural development in ascidians inferred from rDNA sequences. Journal of molecular evolution 40, 413-427.

Hobert, O., 2008. Regulatory logic of neuronal diversity: terminal selector genes and selector motifs. Proceedings of the National Academy of Sciences 105, 20067-20071.

Horie, T., Shinki, R., Ogura, Y., Kusakabe, T.G., Satoh, N., Sasakura, Y.J.N., 2011. Ependymal cells of chordate larvae are stem-like cells that form the adult nervous system. 469, 525.

Huber, J.L., da Silva, K.B., Bates, W.R., Swalla, B.J., 2000. The evolution of anural larvae in molgulid ascidians. Seminars in Cell \& Developmental Biology 11, 419-426.

Ikuta, T., Saiga, H., 2007. Dynamic change in the expression of developmental genes in the ascidian central nervous system: revisit to the tripartite model and the origin of the midbrain-hindbrain boundary region. Dev Biol 312.

Imai, K.S., Stolfi, A., Levine, M., Satou, Y., 2009. Gene regulatory networks underlying the compartmentalization of the Ciona central nervous system. Development 136, 285-293.

Jeffery, W.R., Swalla, B.J., 1991. An evolutionary change in the muscle lineage of an anural ascidian embryo is restored by interspecific hybridization with a urodele ascidian. Developmental biology 145, 328-337.

Jiang, D., Tresser, J.W., Horie, T., Tsuda, M., Smith, W.C., 2005. Pigmentation in the sensory organs of the ascidian larva is essential for normal behavior. Journal of experimental biology 208, 433-438.

Kim, D., Langmead, B., Salzberg, S.L., 2015. HISAT: a fast spliced aligner with low memory requirements. Nature Methods 12, 357.

Kimura, Y., Okamura, Y., Higashijima, S.-i., 2006. alx, a zebrafish homolog of Chx10, marks ipsilateral descending excitatory interneurons that participate in the regulation of spinal locomotor circuits. Journal of Neuroscience 26, 56845697.

Kourakis, M., Borba, C., Zhang, A., Newman-Smith, E., Salas, P., Manjunath, B., Smith, W., 2019. Parallel Visual Circuitry in a Basal Chordate. bioRxiv, 514422.

Kratsios, P., Stolfi, A., Levine, M., Hobert, O., 2012. Coordinated regulation of cholinergic motor neuron traits through a conserved terminal selector gene. Nature neuroscience 15, 205.

Kusakabe, T., Swalla, B.J., Satoh, N., Jeffery, W.R., 1996. Mechanism of an evolutionary change in muscle cell differentiation in ascidians with different modes of development. Developmental biology 174, 379-392.
Li, H., Handsaker, B., Wysoker, A., Fennell, T., Ruan, J., Homer, N., Marth, G., Abecasis, G., Durbin, R., 2009. The sequence alignment/map format and SAMtools. Bioinformatics 25, 2078-2079.

Liu, I.S., Ploder, L., Vidgen, D., van der Kooy, D., Kalnins, V.I., Mclnnes, R.R., 1994. Developmental expression of a novel murine homeobox gene (Chx10): evidence for roles in determination of the neuroretina and inner nuclear layer. Neuron 13, 377-393.

Liu, M., Adelman, Z.N., Myles, K.M., Zhang, L., 2014. A Transcriptome PostScaffolding Method for Assembling High Quality Contigs. Computational Biology Journal 2014, 4.

Lowe, E.K., Stolfi, A., 2018. Developmental system drift in motor ganglion patterning between distantly related tunicates. EvoDevo 9, 18.

Lowe, E.K., Swalla, B.J., Brown, C.T., 2014. Evaluating a lightweight transcriptome assembly pipeline on two closely related ascidian species. PeerJ PrePrints.

Maliska, M.E., Pennell, M.W., Swalla, B.J., 2013. Developmental mode influences diversification in ascidians. Biology letters 9, 20130068.

Nishino, A., 2018. Morphology and Physiology of the Ascidian Nervous Systems and the Effectors, Transgenic Ascidians. Springer, pp. 179-196.

Nishino, A., Okamura, Y., Piscopo, S., Brown, E.R., 2010. A glycine receptor is involved in the organization of swimming movements in an invertebrate chordate. BMC neuroscience 11, 6 .

Pasini, A., Amiel, A., Rothbächer, U., Roure, A., Lemaire, P., Darras, S., 2006. Formation of the ascidian epidermal sensory neurons: insights into the origin of the chordate peripheral nervous system. PLoS biology 4, e225.

Patro, R., Duggal, G., Kingsford, C., 2015. Salmon: accurate, versatile and ultrafast quantification from RNA-seq data using lightweight-alignment. Biorxiv, 021592.

Pennati, R., Ficetola, G.F., Brunetti, R., Caicci, F., Gasparini, F., Griggio, F., Sato, A., Stach, T., Kaul-Strehlow, S., Gissi, C., Manni, L., 2015. Morphological Differences between Larvae of the Ciona intestinalis Species Complex: Hints for a Valid Taxonomic Definition of Distinct Species. PLOS ONE 10, e0122879.

Porter, M.L., Crandall, K.A.J.T.i.E., Evolution, 2003. Lost along the way: the significance of evolution in reverse. 18, 541-547.

Pryszcz, L.P., Gabaldón, T., 2016. Redundans: an assembly pipeline for highly heterozygous genomes. Nucleic acids research 44, e113-e113.

Racioppi, C., Valoroso, M.C., Coppola, U., Lowe, E.K., Brown, C.T., Swalla, B.J., Christiaen, L., Stolfi, A., Ristoratore, F., 2017. Evolutionary loss of melanogenesis in the tunicate Molgula occulta. EvoDevo 8, 11.

Rudolf, J., Dondorp, D., Canon, L., Tieo, S., Chatzigeorgiou, M., 2018. Quantitative analysis reveals the basic behavioural repertoire of the urochordate Ciona intestinalis. bioRxiv, 382465.

Ryan, K., Lu, Z., Meinertzhagen, I.A., 2016. The CNS connectome of a tadpole larva of Ciona intestinalis (L.) highlights sidedness in the brain of a chordate sibling. Elife 5.

Ryan, K., Lu, Z., Meinertzhagen, I.A., 2017. Circuit homology between decussating pathways in the Ciona larval CNS and the vertebrate startle-response pathway. Current Biology 27, 721-728.

Ryan, K., Lu, Z., Meinertzhagen, I.A., 2018. The peripheral nervous system of the ascidian tadpole larva: Types of neurons and their synaptic networks. Journal of Comparative Neurology 526, 583-608.

Ryan, K., Meinertzhagen, I.A., 2019. Neuronal identity: the neuron types of a simple chordate sibling, the tadpole larva of Ciona intestinalis. Current opinion in neurobiology 56, 47-60.

Salas, P., Vinaithirthan, V., Newman-Smith, E., Kourakis, M.J., Smith, W.C., 2018. Photoreceptor specialization and the visuomotor repertoire of the primitive chordate Ciona. Journal of Experimental Biology, jeb. 177972.

Sasakura, Y., 2018. Cellulose production and the evolution of the sessile lifestyle in ascidians. Sessile Organisms 35, 21-29. 
bioRxiv preprint doi: https://doi.org/10.1101/567719; this version posted March 5, 2019. The copyright holder for this preprint (which was not certified by peer review) is the author/funder, who has granted bioRxiv a license to display the preprint in perpetuity. It is made available under aCC-BY-NC-ND 4.0 International license.

Lowe et al., March 012019 - preprint copy - BioRxiv

Satou, Y., Takatori, N., Yamada, L., Mochizuki, Y., Hamaguchi, M., Ishikawa, H., Chiba, S., Imai, K., Kano, S., Murakami, S.D., 2001. Gene expression profiles in Ciona intestinalis tailbud embryos. Development 128, 2893-2904.

Shenkar, N., Gittenberger, A., Lambert, G., Rius, M., Moreira Da Rocha, R., Swalla, B.J., Turon, X., 2019. Ascidiacea World Database. Molgula Forbes, 1848. Accessed through: World Register of Marine Species.

Stolfi, A., Gandhi, S., Salek, F., Christiaen, L., 2014a. Tissue-specific genome editing in Ciona embryos by CRISPR/Cas9. Development 141, 4115-4120.

Stolfi, A., Levine, M., 2011. Neuronal subtype specification in the spinal cord of a protovertebrate. Development 138, 995-1004.

Stolfi, A., Lowe, E.K., Racioppi, C., Ristoratore, F., Brown, C.T., Swalla, B.J., Christiaen, L., 2014b. Divergent mechanisms regulate conserved cardiopharyngeal development and gene expression in distantly related ascidians. Elife 3.

Stolfi, A., Wagner, E., Taliaferro, J.M., Chou, S., Levine, M., 2011. Neural tube patterning by Ephrin, FGF and Notch signaling relays. Development 138, 5429-5439.

Swalla, B.J., Jeffery, W.R., 1990. Interspecific hybridization between an anural and urodele ascidian: differential expression of urodele features suggests multiple mechanisms control anural development. Developmental biology 142, 319-334.

Swalla, B.J., Jeffery, W.R., 1992. Vestigial brain melanocyte development during embryogenesis of an anural ascidian. Development, growth \& differentiation 34, 17-25.

Takahashi, T., Holland, P.W., 2004. Amphioxus and ascidian Dmbx homeobox genes give clues to the vertebrate origins of midbrain development. Development 131, 3285-3294.

Takamura, K., Minamida, N., Okabe, S., 2010. Neural map of the larval central nervous system in the ascidian Ciona intestinalis. Zoological science 27, 191203.

Torrence, S.A., Cloney, R.A.J.Z., 1982. Nervous system of ascidian larvae: caudal primary sensory neurons. 99, 103-115.

Tsuda, M., Sakurai, D., Goda, M., 2003. Direct evidence for the role of pigment cells in the brain of ascidian larvae by laser ablation. Journal of Experimental Biology 206, 1409-1417.

Vorontsova, M., Nezlin, L., Meinertzhagen, I.J.A.Z., 1997. Nervous system of the larva of the ascidian Molgula citrina (Alder and Hancock, 1848). 78, 177-185.

Yagi, K., Makabe, K., 2001. Isolation of an early neural maker gene abundantly expressed in the nervous system of the ascidian, Halocynthia roretzi. Development genes and evolution 211, 49-53.

Zega, G., Thorndyke, M.C., Brown, E.R., 2006. Development of swimming behaviour in the larva of the ascidian Ciona intestinalis. Journal of experimental biology 209, 3405-3412.

Zeller, R.W., 2018. Electroporation in Ascidians: History, Theory and Protocols, Transgenic Ascidians. Springer, pp. 37-48. 